Introduction Non-alcoholic fatty liver disease (NAFLD) is the leading liver disease in Europe and North America. 30\% of patients with NAFLD are estimated to develop inflammatory non-alcoholic steatohepatitis (NASH) with the potential to lead to cirrhosis and hepatocellular carcinoma. Current evidence suggests that gut bacterial products can drive hepatic inflammation by activating specific innate pattern recognition receptors (PRRs) such as TLR4 and the NALP3 inflammasome. Both receptors are expressed by liver resident macrophages, Kupffer cells (KCs).

Methods To determine the role of KCs and interactions with PRRs in NASH, mice were fed a methionine choline deficient (MCD) diet for three weeks to induce NASH. Liposomal clodronate was used to deplete KCs. Serum ALT levels were measured and hepatic inflammatory infiltrates characterised by flow cytometry. Real-time qPCR was used to assess changes in gene expression. Murine findings were correlated with human liver tissue from NASH patients. Groups were compared by one-way ANOVA and significance set at $\mathrm{P}<0.05$.

Results NALP3 KO (knock-out), TLR4 KO or KC deficient WT mice on a MCD diet developed reduced liver damage and decreased T lymphocyte recruitment compared to WT MCD controls. Combined KC depletion and NLRP3 KO however lead to significantly worse liver injury and progressive fibrosis as measured by collagen expression. Further investigation revealed an as yet unrecognised role for KC expressed NALP6 activation which mediated antiinflammatory responses and modulation of hepatic IL-22 responsiveness to reduce liver injury.

Conclusion

1. KC activation through NALP3 and TLR4 increases hepatic inflammation in the MCD model of NASH.

2. KCs have a dual role in NASH as they also express NALP6 with anti-inflammatory properties and are able to reduce hepatic injury through modulation of IL-22 responsiveness modulation.

Disclosure of Interest None Declared

\section{Endoscopy free papers}

\section{OC-009 ENDOSCOPIC THERAPY FOR ZENKER'S DIVERTICULUM - A "BRIDGE" TOO FAR FOR GASTROENTEROLOGISTS?}

doi:10.1136/gutjnl-2013-304907.009

1."M Smith, 'M Widlak, 'N Molony, 'S Ishaq. 'Dudley Group of Hospitals NHS Foundation Trust, NHS, Dudley, UK

Introduction Zenker's diverticulum, caused by dysfunction of the cricopharyngeal (CP) muscle, is a disease of the elderly causing dysphagia. Although rigid endoscopic CP myotomy is the treatment of choice, flexible endoscopic therapy is another technique available. It is performed under sedation without need for anaesthetic or neck extension, a key advantage in elderly patients with significant comorbidity.
Methods We describe the first UK experience of endoscopic CP myotomy for Zenker's diverticulum.

Results 4 patients ( 3 male) were referred, aged 74, 73, 60, 80 respectively, with proven Zenker's diverticulum on barium radiology and characteristic symptomatology. Zenker length was $7.0,4.0,2.5,2.5 \mathrm{~cm}$ respectively. 2 patients were refused surgery due to cardiovascular comorbidity, 1 due to limited neck extension with 1 case personal preference. The procedure was performed using propofol sedation. The muscle bridge was cut by hook knife electrocautery alone (3) or with Argon (1). A supplementary clip was applied post electrocautery in 1 case. All patients were observed for 24 hours for signs of perforation and then discharged. There were no complications.

At 4 weeks 3 patients had complete resolution of their symptoms, 1 with marked improvement. Follow up time to date 3.4.7.11 months respectively. There was no reported recurrence of symptoms.

Conclusion Diverticulotomy with a flexible endoscope is an effective treatment for Zenker's diverticulum. It is a relatively simple yet under-utilised technique that avoids general anaesthesia in elderly/ high-risk patients.

Disclosure of Interest None Declared

\section{OC-010 LARGE COHORT STUDY EVALUATING THE ROLE OF HYBRID ESD (H-ESD) AND CONVENTIONAL PIECEMIEAL EMR TECHNIQUE IN THE RESECTION OF LARGE AND CHALLENGING COLONIC POLYPS DEMONSTRATES NO OUTCOME BENEFIT OF H-ESD OVER EMR}

doi:10.1136/gutjnl-2013-304907.010

'R Bhattacharyya, 'P Basford, 'S Tholoor, ' $G$ Longcroft-Wheaton, 1,* $\mathrm{P}$ Bhandari. ${ }^{1}$ Gastroenterology, Portsmouth Hospitals NHS Trust, Portsmouth, UK

Introduction The learning curve for ESD in the west is very long, so a hybrid technique has been proposed. The impact of Hybrid ESD (H-ESD) technique on clinical outcome is unclear. We aim to compare the outcome benefits of Multi-piece EMR and H-ESD in the resection of challenging colonic polyps.

Methods A Prospective cohort study of endoscopic resection of difficult colonic polyps. Patients were tertiary referrals from experienced endoscopists. EMR was defined as submucosal injection followed by piecemeal snare resection. H-ESD involved submucosal injection before mucosal incision with an ESD knife followed by snare resection of the lesion. Endoscopic follow up was performed. Multiple linear regression analysis was performed using SPSS.

Results $347 \mathrm{flat} / \mathrm{sessile} \mathrm{polyps}>20 \mathrm{~mm}$ were resected between 2007-12. Mean follow-up was 1004 days.

H-ESD Cohort $\mathrm{N}=110 / 347(32 \%)$. Mean size was $45 \mathrm{~mm}$ (range 10-170). 25/110(23\%) were salvage procedures for scarred lesions due to failed EMR attempts by other endoscopists. Endoscopic clearance was achieved in $95.5 \%$ of procedures. Need for surgery $(n=4)$ : 1 for perforation and 3 for unexpected cancer. 98.6\% showed no evidence of recurrence at endoscopic follow up.

EMR cohort $\mathrm{N}=237 / 347(68 \%)$. Mean size was $42 \mathrm{~mm}$ (range 20-150). 11/237(4.6\%) were salvage procedures for polyps with

Abstract 0C-010 Table

\begin{tabular}{|c|c|c|c|c|c|c|c|c|}
\hline & SIZE & & TECHNIQUE & & PREV EMR & & SITE & \\
\hline \multirow{2}{*}{$\begin{array}{l}\text { COMPLICATIONS } \\
\text { (25) }\end{array}$} & $15 / 271(5.5 \%)$ & $\begin{array}{l}8 / 76 \\
(10.5 \%)\end{array}$ & $11 / 110(10 \%)$ & $12 / 237(5 \%)$ & $3 / 36(8 \%)$ & $\begin{array}{l}20 / 311 \\
(6 \%)\end{array}$ & $20 / 250(8 \%)$ & $3 / 97(3 \%)$ \\
\hline & $P=0.016$ & & $P=0.11$ & & $P=0.521$ & & $P=0.201$ & \\
\hline $\begin{array}{l}\text { RECURRENCE } \\
\text { (35) }\end{array}$ & $P=0.0001$ & & $P=0.156$ & & $P=0.0001$ & & $P=0.105$ & \\
\hline
\end{tabular}


scarring. Endoscopic clearance was achieved in $93 \%$ of cases. Need for surgery $(n=21)$ : 1 patient for incomplete resection \& 20 for suspicion of cancer in EMR specimen. At endoscopic follow up $98 \%$ of cases had achieved complete clearance.

Risk of recurrence was associated with lesion size $>50 \mathrm{~mm} \&$ scarring due to previous EMR attempts. This was unaffected by technique (EMR or H-ESD). Perforation/microperforation was more likely in the H-ESD Cohort, but the overall complication rates were similar for H-ESD and EMR cohort.

Conclusion Both techniques achieved an excellent overall cure rate for large \& challenging polyps. This is the first large series comparing the two techniques and demonstrates that polyp cure rate was equally good with both techniques. H-ESD technique was used more commonly for polyps with significant scarring \& was associated with slightly higher perforation rates. Our data does not demonstrate any significant outcome benefit of $\mathrm{H}$-ESD technique over the conventional EMR technique.

Disclosure of Interest None Declared

\section{OC-011 BEWARE THE CAECUM: COLONOSCOPIC ADVERSE EVENTS IN THE ENGLISH NHS BOWEL CANCER SCREENING PROGRAMME}

doi:10.1136/gutjnl-2013-304907.011

1." M D Rutter, ${ }^{2} \mathrm{C}$ Nickerson, ${ }^{2} \mathrm{~J}$ Patnick, ${ }^{3} \mathrm{C} \mathrm{J}$ Rees, ${ }^{4} \mathrm{R}$ G Blanks work undertaken on behalf of the NHS BCSP Evaluation Group. 'Gastroenterology, University Hospital of North Tees, Stockton on Tees; ${ }^{2}$ NHS Cancer Screening Programmes, Sheffield; ${ }^{3}$ Gastroenterology, South Tyneside NHS Foundation Trust, South Shields; ${ }^{4}$ Cancer Epidemiology Unit, Oxford University, Oxford, UK

Introduction The NHS bowel cancer screening programme (BCSP) commenced in 2006 and is one of the world's largest organised screening programmes. Minimising adverse events (AEs) is an essential part of the programme. This study examines colonoscopic AEs and asociated risk factors.

Methods The study examined the AEs of bleeding, post-procedure pain and perforation on all colonoscopies conducted between Aug 2006 and Jan 2012 within the BCSP. Crude AE rates were reported and logistic regression used to examine the risk of an $\mathrm{AE}$ against various explanatory variables, including age, gender, polyp size, morphology, location and therapy device. Detailed analysis was conducted on the subset of single polypectomy procedures.

Results Data are from 130,831 colonoscopies including 167,208 polypectomies. There were 30,896 single polypectomy procedures. Caecal polyp location (but not the rest of the proximal colon) and increasing polyp size were the two most important factors predicting risk of bleeding, bleeding requiring transfusion, post-procedure pain and perforation. After adjustment for polyp size the RR for bleeding requiring transfusion for caecal diathermy snare polypectomy was 13.5 (95\%CI 4.5-40.6) relative to the distal colon. After adjustment for polyp size the RR for perforation occurring during caecal non-pedunculated polypectomy was 7.7 (95\%CI $1.3-46.1$ ) relative to the distal colon. The predicted risk of bleeding requiring transfusion for a $50 \mathrm{~mm}$ caecal polypectomy was as high as 1 in 22 . Further analysis applying estimated risks from the single polyp analysis to multiple polypectomies showed that the absolute risk was not additive.

Conclusion This national study is the largest to focus on polypspecific risk factors. Uniquely, we have been able to focus on colonoscopies where only one polypectomy was performed, allowing a detailed examination of risk to be undertaken where the characteristics of the polyp causing the AE can be clearly established. We have confirmed that the greatest risk factor for an $\mathrm{AE}$ is the size of the polyp.

Perhaps more importantly however, for the first time we have demonstrated a substantially and significantly increased risk for both bleeding requiring transfusion and perforation from caecal polyps (but not in other sites in the proximal colon) for a given polyp size, when compared to the distal colon. Endoscopists should take particular care in resecting caecal polyps.

Additional polypectomies, adjusted for polyp size and location, carry a much lesser risk and therefore confirm that performing multiple polypectomies during the same procedure maximises benefit whilst incurring a lesser level of harm per polyp resected.

Disclosure of Interest None Declared

\section{OC-012 HOW COMMONLY IS OESOPHAGEAL CANCER MISSED AT ENDOSCOPY (A UK PRIMARY CARE BASED STUDY)?}

doi:10.1136/gutjnl-2013-304907.012

1,"D Cheung, ${ }^{2}$ S Menon, 'N Trudgill. 'Department of Gastroenterology, Sandwell General Hospital, West Bromwich; 'Department of Gastroenterology, New Cross Hospital, Wolverhampton, UK

Introduction The frequency of upper gastrointestinal cancer being diagnosed following a negative oesophagogastroduodenoscopy (OGD) up to 3 years previously in selected single unit series ranges from $6.7-14 \%$. We have examined how often oesophageal cancer (OC) is missed at OGD in a large unselected primary care cohort and associated risk factors

Methods All subjects with OC from a UK primary care database (The Health Improvement Network (THIN)) were studied. THIN covers over 6 million patients and is regionally and demographically representative of the UK population. A nested case-control study was performed with cases of 'missed OC' subjects who underwent OGD 1-5 years prior to their OC diagnosis and controls subjects who did not have OGD 1-5 years prior to their OC diagnosis. Logistic regression analysis was used to study associations with having an OGD that potentially missed OC.

Results 5354 subjects with OC were identified (3561 (67\%) male, mean age $71 \pm 11$ years) and 9187 OGD were analysed. 380 subjects (237 (62\%) male, mean age $72 \pm 11$ years) had OGD 1-5 years prior to OC diagnosis (715 OGD, mean 1.9 per subject). 266 (5.0\%) subjects had OGD 1-3 years and 144 (2.7\%) had OGD 3-5 years prior to OC diagnosis. $180(47.4 \%)$ of OGD which did not diagnose OC 1-5 years previously had an abnormality detected (Oesophagus - Barrett's 59 (15.5\%), hiatus hernia 45, oesophagitis 16 , gastro-oesophageal reflux disease 17 , stricture 8 , ulcer 7 ; Stomach - gastritis 25, ulcer 7, polyp 4; Duodenum - duodenitis 17 , ulcer 8 , coeliac disease 1 ). The 'missed OC' subjects had a total 329 primary care consultations 1 year prior to their OGD that missed OC. 'Alarm symptoms' were present at 139 consultations (38 anaemia, 1 abdominal mass, 78 dysphagia, 20 haematemesis/ melaena and 6 weight loss). Subjects presenting with anaemia (OR 2.05, 95\%CI 1.4-3.1, p = 0.0006), haematemesis/melaena $(1.82,1.1-3.2, p=0.03)$ or dyspepsia $(2.20,1.7-2.8, p<0.05)$ were more likely to have had an OGD that missed OC. In contrast, subjects with dysphagia $(0.74,0.5-0.9, \mathrm{p}=0.04)$ or weight loss $(0.39,0.2-0.9, p=0.02)$ were less likely to have an OGD that missed OC. Logistic regression did not find any association between age $(0.7,-0.5-1.8, p=0.3)$ or female gender $(1.2,0.9-1.5$, $\mathrm{p}=0.07)$ with OGD that failed to diagnose OC $1-5$ years prior to OC diagnosis.

Conclusion Missing OC at OGD was relatively uncommon in this unselected cohort with $5 \%$ of OC subjects having had an OGD $1-3$ years prior to diagnosis that did not diagnose OC. Presenting with anaemia, haematemesis/melaena or dyspepsia were positively associated with missing OC at OGD. In contrast, subjects with dysphagia or weight loss were less likely to have OC missed at OGD. Many of the OGD which missed OC had an abnormality recorded, with Barrett's oesophagus reported in $15.5 \%$.

Disclosure of Interest None Declared 